This item was submitted to Loughborough's Research Repository by the author.

Items in Figshare are protected by copyright, with all rights reserved, unless otherwise indicated.

\title{
Experimental study of a flexible and environmentally stable electroadhesive device
}

PLEASE CITE THE PUBLISHED VERSION

https://doi.org/10.1063/1.4995458

\section{PUBLISHER}

AIP Publishing (@) Author(s).

VERSION

VoR (Version of Record)

\section{PUBLISHER STATEMENT}

This work is made available according to the conditions of the Creative Commons Attribution 4.0 International (CC BY 4.0) licence. Full details of this licence are available at: http://creativecommons.org/licenses/ by/4.0/

\section{LICENCE}

CC BY-NC-ND 4.0

\section{REPOSITORY RECORD}

Guo, Jianglong, Thomas Bamber, Jagpal Singh, D. Manby, Paul A. Bingham, Laura M. Justham, Jon N. Petzing, Jacques Penders, and Michael R. Jackson. 2019. "Experimental Study of a Flexible and Environmentally Stable Electroadhesive Device”. figshare. https://hdl.handle.net/2134/28033. 


\section{Experimental study of a flexible and environmentally stable electroadhesive device}

J. Guo, T. Bamber, J. Singh, D. Manby, P. A. Bingham, L. Justham, J. Petzing, J. Penders, and M. Jackson

Citation: Appl. Phys. Lett. 111, 251603 (2017);

View online: https://doi.org/10.1063/1.4995458

View Table of Contents: http://aip.scitation.org/toc/apl/111/25

Published by the American Institute of Physics

\section{Articles you may be interested in}

Chemical vapour deposition of freestanding sub-60 nm graphene gyroids

Applied Physics Letters 111, 253103 (2017); 10.1063/1.4997774

Large-scale broadband absorber based on metallic tungsten nanocone structure

Applied Physics Letters 111, 251102 (2017); 10.1063/1.5004520

Electrically driven quantum light emission in electromechanically tuneable photonic crystal cavities

Applied Physics Letters 111, 251101 (2017); 10.1063/1.5008590

Sub-monolayer quantum dot quantum cascade mid-infrared photodetector

Applied Physics Letters 111, 251104 (2017); 10.1063/1.5011239

Double Fe-impurity charge state in the topological insulator $\mathrm{Bi}_{2} \mathrm{Se}_{3}$

Applied Physics Letters 111, 251601 (2017); 10.1063/1.5002567

Enhanced recoverable energy storage density and high efficiency of $\mathrm{SrTiO}_{3}$-based lead-free ceramics Applied Physics Letters 111, 253903 (2017); 10.1063/1.5000980

\section{Scilight}

Sharp, quick summaries illuminating the latest physics research

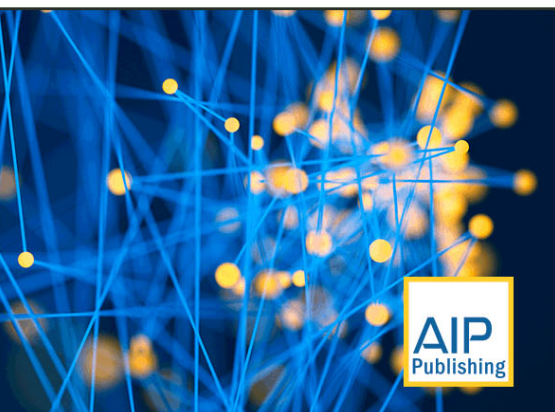




\title{
Experimental study of a flexible and environmentally stable electroadhesive device
}

\author{
J. Guo, ${ }^{1, a)}$ T. Bamber, ${ }^{1}$ J. Singh, ${ }^{2}$ D. Manby, ${ }^{3}$ P. A. Bingham, ${ }^{2}$ L. Justham, ${ }^{1, a)}$ \\ J. Petzing, ${ }^{1}$ J. Penders, ${ }^{2}$ and M. Jackson ${ }^{1}$ \\ ${ }^{1}$ The Wolfson School of Mechanical, Electrical and Manufacturing Engineering, Loughborough University, \\ Loughborough, Leicestershire LE11 3TU, United Kingdom \\ ${ }^{2}$ Materials and Engineering Research Institute, Sheffield Hallam University, City Campus, Sheffield S1 1WB, \\ United Kingdom \\ ${ }^{3}$ Aylesbury Automation Limited, Aylesbury HP20 1DQ, United Kingdom
}

(Received 11 July 2017; accepted 6 December 2017; published online 18 December 2017)

\begin{abstract}
Electroadhesion is a promising adhesion mechanism for robotics and material handling applications due to several distinctive advantages it has over existing technologies. These advantages include enhanced adaptability, gentle/flexible handling, reduced complexity, and ultralow energy consumption. Unstable electroadhesive forces, however, can arise in ambient environments. Electroadhesive devices that can produce stable forces in changing environments are thus desirable. In this study, a flexible and environmentally stable electroadhesive device was designed and manufactured by conformally coating a layer of barium titanate dielectric on a chemically etched thin copper laminate. The results, obtained from an advanced electroadhesive "normal force" testing platform, show that only a relative difference of 5.94\% in the normal force direction was observed. This was achieved when the relative humidity changed from $25 \%$ to $53 \%$, temperature from $13.7^{\circ} \mathrm{C}$ to $32.8^{\circ} \mathrm{C}$, and atmospheric pressure from $999 \mathrm{hPa}$ to $1016.9 \mathrm{hPa}$. This environmentally stable electroadhesive device may promote the application of the electroadhesion technology. (C) 2017 Author(s). All article content, except where otherwise noted, is licensed under a Creative Commons Attribution (CC BY) license (http://creativecommons.org/licenses/by/4.0/).

https://doi.org/10.1063/1.4995458
\end{abstract}

Electroadhesion $^{1}$ is a promising and potentially revolutionary adhesion mechanism for mobile robotics and materials handling applications. Examples of where electroadhesion is in use include electrostatic chucks, ${ }^{2}$ electroadhesive grasping of delicate objects ${ }^{3}$ and fibrous materials, ${ }^{4}$ climbing, ${ }^{5}$ and perching $^{6}$ robots. This is due to certain advantages that electroadhesion has over other adhesion mechanisms such as magnetic, pneumatic, and bio-inspired adhesion methods. ${ }^{7}$ These advantages include enhanced adaptability, gentle/flexible handling, reduced complexity, and ultra-low energy consumption. ${ }^{8}$

Electroadhesion is an electrically controllable and dynamic $^{8}$ electrostatic attraction between an electroadhesive pad and a substrate, with 33 variables influencing the interfacial electroadhesive force. ${ }^{9}$ These variables include voltage $\mathrm{e}^{10,11}$ and material properties such as relative permittivity, ${ }^{10-12}$ pad geometry, ${ }^{13,14}$ surface texture, ${ }^{15}$ and environmental conditions. ${ }^{9,16} \mathrm{~A}$ typical electroadhesion system contains four main parts, including an electroadhesive pad, a power supply, a control system, and a substrate, as can be seen in Fig. 1. The electroadhesive force is generated by high-electric-field induced polarization or electrostatic induction depending on the material type. ${ }^{7}$

Environmental conditions can cause material properties (such as dielectric strength, permittivity, and resistivity) to change and therefore also the polarization properties. Among the environmental factors, Monkman ${ }^{17}$ concluded that only temperature and humidity have a noticeable effect on the electroadhesive force. Savioli et al. ${ }^{18}$ discovered that

\footnotetext{
${ }^{\text {a) }}$ Authors to whom correspondence should be addressed: guojianglong9085@ sina.com and L.Justham@lboro.ac.uk.
}

different shear forces were obtained over three different days with differing temperature and humidity profiles. Guo et al. ${ }^{9}$ demonstrated that unstable normal electroadhesive forces were achieved in different ambient environments. Such variability may hinder the wider application of electroadhesion technologies. An environmentally stable electroadhesive device is, therefore, needed and defined here as an electroadhesive device that can produce stable electroadhesive forces in changing temperature, relative humidity, and ambient pressure profiles.

To address the environmental instability of current electroadhesive pads, Guo et al. ${ }^{16}$ implemented an environmental adaptive electroadhesive actuator that could produce robust electroadhesive forces at different humidity levels. An empirical relationship between the minimum voltages needed to grip a known material and varying humidity levels, however, must be obtained and embedded in the control loop for the electroadhesive end effector to adjust the voltage output for different humidity levels. If a humidity level is not previously included in the empirical relationship, the end effector will not be able to output the right voltage to successfully grip the material. ${ }^{15}$ It has to be noted that various

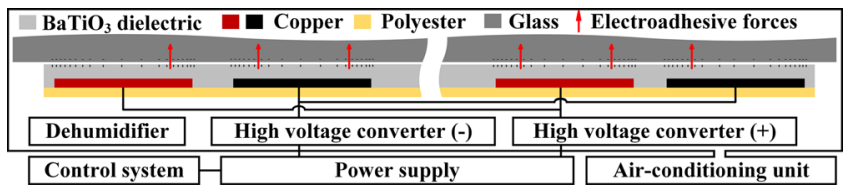

FIG. 1. Schematic diagram of a typical electroadhesion system. 
experiments, however, are needed to obtain reasonably robust and comprehensive empirical relationships between the applied voltage and environmental factors (such as temperature, relative humidity, and ambient pressure) to calibrate the voltage adjustment based on the information feed from the sensors, which is not cost-effective.

An alternative method is to coat electroadhesive pads with materials that are less sensitive to environmental variations, thus producing stable electroadhesive properties in changing environments. In this way, the troublesome compensation/calibration procedure aforementioned can be eliminated. There is, however, little research concerning coating electroadhesive pads with materials that are less sensitive to environmental variations, thus producing stable electroadhesive properties in changing environments.

This study reports the development and experimental characterization of an environmentally stable electroadhesive pad. Previous studies showed that improved performance, including greater adhesive forces and quicker release characteristics, was achieved by using a barium titanate $\left(\mathrm{BaTiO}_{3}\right)$ coated electroadhesive pad. ${ }^{19}$ Inspired by the fact that the dielectric constant of $\mathrm{BaTiO}_{3}$ changes little in different ambient environments, ${ }^{19}$ the studied environmentally stable, flexible, and lightweight electroadhesive pad was made from a chemically etched thin copper laminate (UK Insulations Ltd., UK) and a $\mathrm{BaTiO}_{3}$ dielectric (dielectric constant of 8, Applied Technologies, Inc., US). The copper laminate was a $20 \mu \mathrm{m} / 23 \mu \mathrm{m}$ copper polyethyleneterephthalate (PET) bilayer.

The flexible and environmentally stable electroadhesive pad manufacturing process consists of five major steps. These steps are similar to the electroadhesive pad manufacture procedures published previously ${ }^{14}$ except that the dielectric coating and degassing/curing method were different. First, an A4 sized $43-\mu \mathrm{m}$-thick copper-PET sheet was thoroughly cleaned and dried. A worm-comb geometry was then printed onto the copper side using a solid-ink (wax) printer (Xerox UK Ltd., UK). The worm-comb geometry was selected due to the fact that a previous experimental comparison of different geometries concluded that the worm-comb shape could bring slightly greater adhesive forces. ${ }^{14}$ The dielectric strength and permittivity of the PET were $310 \mathrm{kV} / \mathrm{mm}$ and 3.2 , respectively. The electrode width and gap were both $3.6 \mathrm{~mm}$. The effective pad area was $180 \mathrm{~mm} \times 198 \mathrm{~mm}$. Second, the wax printed copper laminate was chemically etched, thoroughly cleaned, and dried. For the third stage, the laminate was clamped on a $10 \mathrm{~mm}$ aluminium pad holder. A $50-\mu$ m-thick $\mathrm{BaTiO}_{3}$ dielectric was then doctor-blade coated on the etched laminate using a micrometer adjustable film applicator (MTI Corporation, US) in a spray booth.

The $S q$ (root mean square height of the surface) value of the dielectric surface was $0.24 \pm 0.01 \mu \mathrm{m}$. To measure this, an Alicona InfiniteFocus G4 (Alicona, Austria) was used, with a $\times 20$ objective. 10 different areas of the dielectric surface were measured. Surface texture information of one area is presented in Fig. 2(a). The $\mathrm{BaTiO}_{3}$ dielectric was made of $\mathrm{BaTiO}_{3}$ particles suspended in fluoropolymer. A FE-SEM (field emission scanning electron microscopy, JSM-7800F, JEOL, Japan) image of the dielectric subsurface is shown in
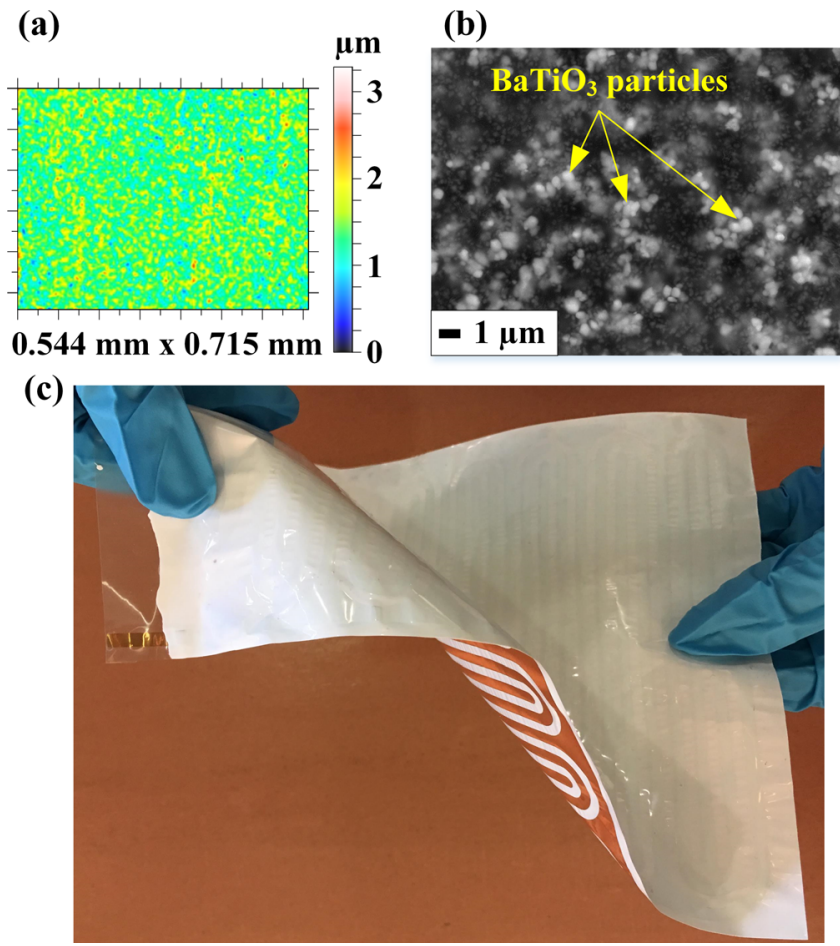

FIG. 2. The $\mathrm{BaTiO}_{3}$ dielectric coated electroadhesive pad: (a) surface texture information (form removed) of one area of the $\mathrm{BaTiO}_{3}$ dielectric surface, (b) SEM image of the dielectric, and (c) the proposed flexible and environmentally stable electroadhesive pad.

Fig. 2(b). The detector (X-Max 80, Oxford Instruments, UK) voltage and current were $20 \mathrm{kV}$ and $4.3 \mathrm{nA}$, respectively. For the fourth stage, the coated electroadhesive pad and pad holder were placed in a vacuum oven (Fistreem International Ltd., UK) for degassing at room temperature for $10 \mathrm{~min}$ followed by curing at $120^{\circ} \mathrm{C}$ for $20 \mathrm{~min}$. Finally, the electroadhesive pad was taken out of the oven and allowed to cool at room temperature for $24 \mathrm{~h}$ before testing. The prototype of the manufactured flexible and environmentally stable electroadhesive pad can be seen in Fig. 2(c).

Normal electroadhesive forces were measured using the advanced force measurement test rig and procedure published previously. ${ }^{9,10}$ The force tests were conducted over three days, and each test was repeated five times. A 15-time test was conducted previously to check the repeatability of the testing procedure and concluded that five times were enough to produce robust and repeatable results. It has to be noted that, however, these tests should be conducted under the condition that there is no degradation and dielectric breakdown of the electroadhesive pad. An air conditioner (Ecoair London, UK) and a dehumidifier (Ecoair London, UK), enclosed in an insulated foam sealed environmental chamber, were used to produce varying and controlled environmental conditions. As shown in Fig. 3, over the three days, the relative humidity changed from $25 \%$ to $53 \%$. The room temperature changed from $13.7^{\circ} \mathrm{C}$ to $32.8^{\circ} \mathrm{C}$. The atmospheric pressure changed from $999 \mathrm{hPa}$ to $1016.9 \mathrm{hPa}$. Two high voltage converters (EMCO High Voltage Corporation, US), connected to a 0-5 V reference input DC power supply, were used to provide $3.6 \mathrm{kV}$ to energize the electroadhesive pad. The electroadhesive pad was charged for $90 \mathrm{~s}$ before measuring the electroadhesive force between 


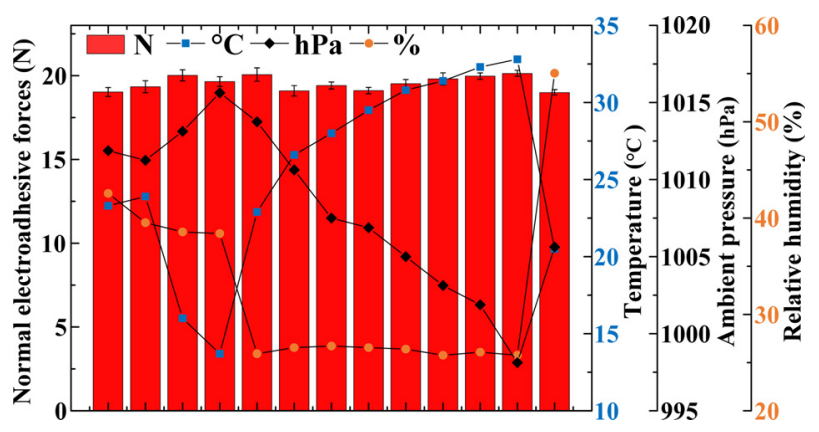

FIG. 3. Measured normal electroadhesive forces under changing environments over three days.

the $\mathrm{BaTiO}_{3}$ dielectric side of the pad and a glass plate $(S q=2.4 \pm 0.2 \mathrm{~nm})$. The maximum normal electroadhesive force recorded was $20.14 \pm 0.18 \mathrm{~N}$, whereas the minimum was $19.01 \pm 0.16 \mathrm{~N}$.

In this study, a relative difference of only $5.94 \%$ was observed in the electroadhesive forces when the sample was exposed to a temperature difference of $19.1^{\circ} \mathrm{C}$, a relative humidity difference of $28 \%$, and an atmospheric pressure difference of $17.9 \mathrm{hPa}$, as shown in Fig. 3. Please note that the relative difference is defined here as (maximum - minimum)/ minimum $\times 100 \%$.

The microstructures of the dielectric surface would influence the electroadhesive force obtainable. ${ }^{14}$ As the aim of this study is to obtain the relationship between the electroadhesive force and different environmental conditions, the surface microstructure of the proposed electroadhesive pad should be fixed. The same electroadhesive pad should, therefore, be used to eliminate the variability of surface microstructures. However, in order to show that the thickness of the dielectric layer will not affect the stable functional behavior of the proposed electroadhesive pad, a thinner $(30 \mu \mathrm{m}) \mathrm{BaTiO}_{3}$ dielectric coated electroadhesive pad was manufactured and tested at the same time. The results of this thinner electroadhesive pad showed that a relative difference of $6.13 \%$ was obtained in the electroadhesive forces under the same environmental conditions aforesaid. The result was close to the $50 \mu \mathrm{m}$ one. However, further tests using other coating thicknesses are needed to confirm that similar performance can be achieved using different coating thicknesses.

In this study, as the glass substrate was placed underneath the electroadhesive pad, the electroadhesive force was induced mainly by electric polarization. The electroadhesive force was measured when the $\mathrm{BaTiO}_{3}$ dielectric was facing the substrate. Previous results showed that the dielectric constant of $\mathrm{BaTiO}_{3}$ based materials was relatively stable from $10{ }^{\circ} \mathrm{C}$ to $50{ }^{\circ} \mathrm{C}$. ${ }^{20} \mathrm{In}$ addition, the variation of the dielectric constant of $\mathrm{BaTiO}_{3}$ based materials was relatively small from $10 \%$ to $50 \% .^{21}$ As shown in Fig. 3, the relative difference in the electroadhesive force obtained was relatively small. It was assumed that this was due to the fact the coated $\mathrm{BaTiO}_{3}$ dielectric's material properties were quite stable in a range of ambient environments, ${ }^{20,21}$ thus producing relatively stable electroadhesive forces. In order to experimentally verify this, two other electroadhesive pads, manufactured after the second manufacturing stage aforementioned, with the same electrode geometry and dimensions, were tested by facing the bare electrode

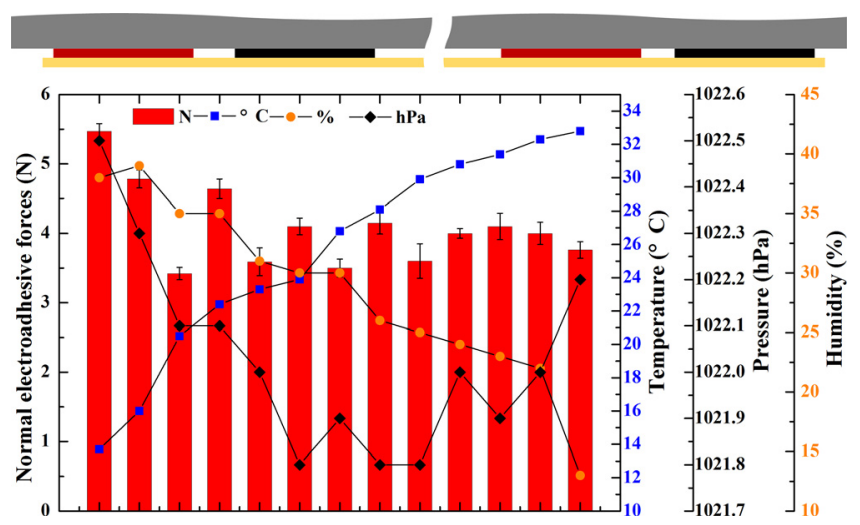

FIG. 4. The measured adhesive forces when the bare electrode side of the electroadhesive pad was facing the glass substrate.

(electroadhesive pad coated with nothing) and PET (electroadhesive pad coated with PET) side towards the glass substrate. For the electroadhesive pad coated with nothing, a relative difference of $59.9 \%$ in the electroadhesive forces was observed when the pad was exposed to a temperature difference of $19.1^{\circ} \mathrm{C}$, a relative humidity difference of $25 \%$, and an atmospheric pressure difference of $0.7 \mathrm{hPa}$, as shown in Fig. 4. For the electroadhesive pad coated with PET, a relative difference of $117.6 \%$ in the electroadhesive forces was obtained when the pad was exposed to a temperature difference of $19.1^{\circ} \mathrm{C}$, a relative humidity difference of $24 \%$, and an atmospheric pressure difference of $0.9 \mathrm{hPa}$, as shown in Fig. 5. However, a more in-depth understanding of the fundamental mechanism behind this should be conducted in the future.

Previous results have shown a relative difference of $195.8 \%$ in the forces for a polyurethane coated electroadhesive pad exposed to a temperature difference of $0.7^{\circ} \mathrm{C}$, a relative humidity difference of $21 \%$, and an atmospheric pressure difference of $19.1 \mathrm{hPa} .{ }^{9}$ Similarly, a relative difference of $41.1 \%$ was observed in the forces for a polyimide coated electroadhesive pad which was exposed to a temperature difference of $1.6^{\circ} \mathrm{C}$, a relative humidity difference of $12 \%$, and an atmospheric pressure difference of $7 \mathrm{hPa} .{ }^{16}$ The reported flexible and environmentally stable electroadhesive device presented here therefore demonstrates a step-change improvement in stability across a wide range of ambient operating conditions. This result suggests that significant

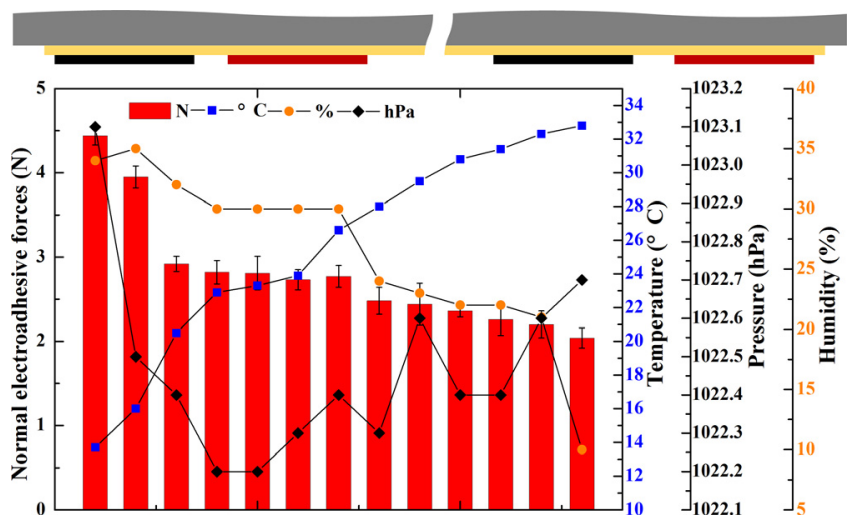

FIG. 5. The measured adhesive forces when the PET side of the electroadhesive pad was facing the glass substrate. 


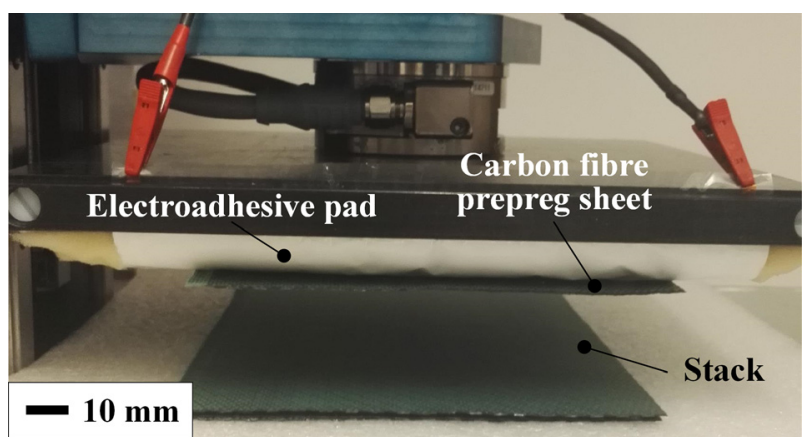

FIG. 6. Electroadhesive grasping of a carbon fibre prepreg sheet from a stack.

improvements in electroadhesive devices may be possible through further investigations into alternative coating materials. This has the potential to greatly improve the capabilities of this adhesion method and increase its use in robotics and material handling applications. One possible industrial application is the robotic pick-and-place of carbon fibre prepreg sheets, as demonstrated in Fig. 6, where a single layer of carbon fibre prepreg sheet was picked up by the electroadhesive gripper from a stack.

The authors acknowledge support from the EPSRC Centre for Innovative Manufacturing in Intelligent Automation, under Grant Reference No. EP/IO33467/1, and support from Innovate UK under Project Reference No. 101549. In addition, the authors acknowledge use of the facilities and the assistance of Dr. Keith Yendal in the Loughborough Materials Characterisation Centre and Mr. Jagpal Singh in the Loughborough Metrology Lab.
${ }^{1}$ K. Rahbek, "Electroadhesion apparatus," U.S. patent 2,025,123 (24 December 1935).

${ }^{2}$ G. Shim and H. Sugai, Plasma Fusion Res. 3, 51 (2008).

${ }^{3}$ J. Shintake, S. Rosset, B. Schubert, D. Floreano, and H. Shea, Adv. Mater. 28(2), 231-238 (2016).

${ }^{4}$ G. J. Monkman, Int. J. Rob. Res. 14(2), 144-151 (1995).

${ }^{5}$ R. Liu, R. Chen, H. Shen, and R. Zhang, Int. J. Adv. Rob. Syst. 10(36), 1-9 (2012).

${ }^{6}$ M. Graule, P. Chirarattananon, S. Fuller, N. Jafferis, K. Ma, M. Spenko, R. Kornbluh, and R. Wood, Science 352, 978-982 (2016).

${ }^{7}$ J. Guo, L. Justham, M. Jackson, and R. Parkin, Key Eng. Mater. 649, 22-29 (2015).

${ }^{8}$ T. Bamber, J. Guo, J. Singh, M. Bigharaz, P. A. Bingham, L. Justham, J. Petzing, J. Penders, and M. Jackson, J. Phys. D: Appl. Phys. 50(20), 205304 (2017).

${ }^{9}$ J. Guo, T. Bamber, M. Chamberlain, L. Justham, and M. Jackson, J. Phys. D: Appl. Phys. 49(41), 415304 (2016).

${ }^{10}$ J. Guo, T. Bamber, J. Pezting, L. Justham, and M. Jackson, Appl. Phys. Lett. 110(5), 051602 (2017).

${ }^{11}$ K. H. Koh, M. Sreekumar, and S. G. Ponnambalam, Materials 7, 4963-4981 (2014).

${ }^{12}$ C. Cao, X. Sun, Y. Fang, Q. Qin, A. Yu, and X. Feng, Mater. Des. 89, 485-491 (2016).

${ }^{13}$ D. Ruffatto, J. Shah, and M. Spenko, J. Electrost. 72(2), 147-155 (2014).

${ }^{14}$ J. Guo, T. Bamber, T. Hovell, M. Chamberlain, L. Justham, and M. Jackson, IFAC-PapersOnLine 49(21), 309-315 (2016).

${ }^{15}$ J. Guo, M. Tailor, T. Bamber, M. Chamberlain, L. Justham, and M. Jackson, J. Phys. D: Appl. Phys. 49(3), 35303 (2016).

${ }^{16}$ J. Guo, T. Bamber, M. Chamberlain, L. Justham, and M. Jackson, IEEE Rob. Autom. Lett. 2(2), 538-545 (2017).

${ }^{17}$ G. J. Monkman, "Electrostatic techniques for fabric handling," M.Sc. thesis, University of Hull, 1987.

${ }^{18}$ L. Savioli, G. Sguotti, A. Francesconi, F. Branz, J. Krahn, and C. Menon, Proc. SPIE 9061, 906129 (2014).

${ }^{19}$ Aylesbury Automation Limited, "Electroadhesive gripper," UK patent application GB1608729.8 (18 May 2016).

${ }^{20}$ D. Sitko, W. Bąk, B. Garbarz-Glos, A. Budziak, C. Kajtoch, and A. Kalvane, IOP Conf. Ser.: Mater. Sci. Eng. 49, 012050 (2013).

${ }^{21}$ J. Wang, B. K. Xu, S. P. Ruan, and S. P. Wang, Mater. Chem. Phys. 78(3), 746-750 (2003) 